\title{
Cardiovascular imaging 2018 in the International Journal of Cardiovascular Imaging
}

\author{
Johan H. C. Reiber ${ }^{1}$. Gabriel Tensol R. Pereira ${ }^{2} \cdot$ Hiram G. Bezerra ${ }^{2} \cdot$ Johan De Sutter $^{3} \cdot$ Paul Schoenhagen $^{4}$. \\ Arthur E. Stillman ${ }^{5} \cdot$ Nico R. L. Van de Veire ${ }^{6}$
}

Published online: 13 March 2019

(c) Springer Nature B.V. 2019

Please, find below an overview of the most relevant papers in the International Journal of Cardiovascular Imaging over the year 2018 for the different modalities.

\section{X-ray imaging}

Detter et al. evaluated in an animal experiment the value of fluorescent cardiac imaging (FCI) for intraoperative qualitative angiographic and quantitative myocardial perfusion assessment during graded coronary artery bypass grafting (CAB) stenosis compared to coronary angiography [1]. They found that only visual assessment of CAB quality using FCI was limited as compared to coronary angiography. However, additional quantitative FCI (QFCI) identified graded $\mathrm{CAB}$ stenosis and occlusion with a significant correlation $(\mathrm{r}=0.955)$ to transit-time flow measurement (TTFM).

The team of Hayiroglu et al. investigated the prognostic value of Syntax Score II in STEMI patients complicated with cardiogenic shock and treated with primary PCI [2]. 492 patients were included in the retrospective analysis and subdivided in three tertiles based on their Syntax Score.

Johan H. C. Reiber

J.H.C.Reiber@lumc.nl

1 Department of Radiology, Division of Image Processing, Leiden University Medical Center, Leiden, The Netherlands

2 Department of Cardiology, Case Western Hospitals, Cleveland, OH, USA

3 Department of Cardiology, AZ Maria Middelares Gent and University Gent, Ghent, Belgium

4 Department of Radiology, The Cleveland Clinic Foundation, Cleveland, OH, USA

5 Department of Radiology, Emory University Hospital, Atlanta, GA, USA

6 Department of Cardiology, AZ Maria Middelares Gent and Free University Brussels, Brussels, Belgium
They found that patients in tertile 3 had a 6.2-fold hazard of in-hospital death, and therefore concluded that the Syntax II Score is a simple, feasible and clinically applicable tool for rapid risk stratification in this patient population [2].

Left atrium (LA) size is a well-studied predictor of atrial fibrillation (AF) recurrence after pulmonary vein isolation (PVI). Strisciuglio et al. was interested in studying the value of three-dimensional rotational angiography (3DRA LA volume index) as a valid alternative to the commonly used transthoracic echocardiography (TTE LA volume index) [3]. For that purpose they analysed 352 consecutive patients with symptomatic paroxysmal or persistent atrial fibrillation referred for PVI. From a multivariate analysis, they found that 3DRA-LAVI was the only independent predictor for AF recurrence [HR 1.01 (1.00-1.03), $\mathrm{p}=0.017]$. And therefore, concluded that left atrial volume measured with 3DRA is superior to TTE assessment and to AF history in predicting atrial fibrillation recurrence after PVI.

In 3D reconstruction of coronary arterial segments from standard X-ray angiography, the definition of the coronary centerline plays an important step in the total reconstruction procedure. Kunio et al. developed and validated a vessel centerline reconstruction algorithm from non-isocentric and non-orthogonal paired monoplane angiographic images [4]. Their method was tested in phantoms, in animal experiments as well in a small number of patients and demonstrated high accuracy and reproducibility.

In this paper Karabağ et al. examined the predictive performance of Syntax II score in in-hospital and long-term mortality of STEMI patients and to compare SYNTAX score (SS) and TIMI risk score (TRS) [5]. The predictive power of SS-II, SS, and TRS were compared by ROC curve and decision curve analysis. SS-II surpassed SS and TRS in long-term and in-hospital mortality prediction. They concluded that SS-II is a powerful tool to predict in-hospital and long-term mortality from all causes in STEMI patients treated with p-PCI. 
Hayıroğlu et al. investigated the femoral hematoma predictive value of angle of sheath to trochanter major in patients undergoing elective coronary procedures in a prospective analysis of 246 patients [6]. Twenty three patients developed a hematoma and the remaining 223 patients functioned as control. The best cut-off value of the angle of sheath to trochanter major to predict femoral hematoma was $15.6^{\circ}$ with $74 \%$ sensitivity and $70 \%$ specificity (AUC: $0.75 ; 95 \%$ CI $0.63-0.86 ; \mathrm{p}<0.001$ ). These data suggests the importance of fluoroscopic guidance during femoral artery access with the predictive role of the angle of sheath to trochanter major.

The aim of the study by Maccagni et al. was to evaluate the impact of the implementation of a noise reduction technology (NRT) on patient radiation dose during CTO PCI [7]. It is well known that CTO procedures are associated with high radiation doses to patients and personnel. The population consisted of 60 patients following the standard protocol and 127 with the NRT approach. They concluded that the use of NRT in CTO PCI is associated with lower patient radiation dose and similar image quality.

Tian et al. aimed to assess the prevalence of anomalous origin of coronary arteries in a Chinese population who underwent coronary angiography for coronary artery disease, and explore any patterns in the common variants and typical anomalies, especially the potentially serious ones [8]. From a total of 110,158 patients included in the study, $0.76 \%$ (835 cases) had anomalous origin of coronary arteries. And $47.54 \%$ (397 cases) of the anomalies were shown to be potentially serious, and an RCA arising from the left sinus of Valsalva (LSV) was the most common subtype (39.28\%, 328 cases). They concluded that more clinical attention should be paid to this condition due to the potential risk of serious sequelae.

The purpose of the study by Her et al. was to compare the efficacy of paclitaxel-coated balloon (PCB) treatment with stent implantation for de novo coronary lesions after successful plain old balloon angioplasty (POBA) guided by fractional flow reserve (FFR) [9]. 200 patients were included in the study and the primary endpoint was late lumen loss at 9 months. The conclusion was that PCB treatment guided by POBA-FFR showed excellent 9 months angiographic and functional results, as well as comparable 12 months clinical outcomes, compared with stent implantation for de novo coronary lesions.

The aim of this meta-analysis carried out by the team of Hideo-Kajita was to examine the available data to determine if complete revascularization (using either angio- or fractional flow reserve guidance-FFR) following primary PCI in STEMI patients without cardiogenic shock impacts clinical outcomes [10]. A total of nine studies (3317 patients) were included. They concluded on the basis of all these data that compared to culprit-only revascularization, treating multi-vessel disease in STEMI patients using FFR guidance is associated with decreased incidence of MACE, due to a decreased rate of revascularization.

Finally, Sonaglioni et al. validated a new modified method for measuring the anthropometric Haller index (HI), obtained without radiological exposure [11]. This index is used to assess chest conformation and pectus excavatum (PE). This new method was based on the use of a rigid ruler and of a $2.5 \mathrm{MHz}$ ultrasound transducer for the assessment of latero-lateral and antero-posterior chest diameters, respectively. They enrolled 100 consecutive patients. They concluded that the modified HI might allow a quick description of the chest conformation without radiological exposure and a more immediate comprehension of its possible influence on the cardiac kinetics and function, as assessed by echocardiography or other imaging modalities.

\section{Intravascular imaging}

The year of 2018 was very exciting for the intravascular imaging field, with several new technologies being investigated in addition of important definitions on stablished modalities. The International Journal of Cardiovascular Imaging covered several of those topics involving all aspects of intravascular imaging.

Highlighting the importance of intravascular imaging, especially OCT, on guiding PCI, Kuku et al. performed a meta-analysis including 2781 patients, from three RCT and three observational studies, comparing PCI guided by intravascular imaging to angiography. They found that OCT guided PCI was associated with reduction in adverse clinical events, including cardiac deaths, MACE composite cardiac deaths, myocardial infarction and repeat revascularization when compared with angiography guidance, but found no difference when comparing OCT to IVUS. We look forward to clinical trials utilizing new metrics on OCT and it's comparison to IVUS on powered studies [12].

\section{Evaluation of bioresorbable vascular scaffolds}

On last year edition of this journal Shen et al. evaluated the safety and feasibility of XINSORB bioresorbable sirolimus-eluting scaffold and this year the same group analyzed the healing score of the scaffold in a 6 months follow-up period. Healing score was proposed as a metric to measure the vessel recovery after stent/scaffold implantation and it is believed to correlate with adverse outcomes including stent thrombosis and restenosis. On this study the authors found that the score for the XINSORB was 6.23 which is similar to what was found at the ABSORB Cohort B1 [13]. 


\section{Evaluation of metallic stents}

Paramount for the stent healing process is having strut coverage without excessive neointimal hyperplasia (NIH). Using OCT and QCA Gil et al. investigated the effect of the stent platform (stainless steel and cobalt-chromium; sirolimus concentration of 1.0 and $1.2 \mu \mathrm{g} / \mathrm{mm}^{2}$ ) and thickness on neointimal formation by utilizing three DES stents covered with similar biodegradable polymer and elute sirolimus but different thickness, material and platform. It was found that the thickness of the struts and the drug concentration are very important on NIH formation, whereas the material did not play a significant role [14].

On a similar topic Kobayashi et al. compared very-early strut coverage and NIH formation between a new biodegradable polymer drug eluting stent (Synergy ${ }^{\mathrm{TM}}$ and Ultimaster ${ }^{\mathrm{TM}}$ ) and a second-generation durable polymer everolimuseluting stent with OCT (Xience ${ }^{\mathrm{TM}}$ and Premier ${ }^{\mathrm{TM}}$ ). Previous studies showed that biodegradable eluting stents were associated with delayed vessel healing and stent endothelization. On a population of 57 patients, the new biodegradable polymer eluting stent had superior NIH thickness $(21.2 \mu \mathrm{m}$ vs. $9.8 \mu \mathrm{m}$ ) and neointimal coverage (53.9\% vs. $28 \%$ ), showing a significant improvement from the previous generation [15].

In an attempt to characterize differences in neoatherosclerosis between first (Cypher $®$ and Taxus $®)$ and second (Xience ${ }^{\circledR}$, Promus ${ }^{\circledR}$, Endeavor ${ }^{\circledR}$, Resolute Integrity ${ }^{\circledR}$ and Nobori $\left.{ }^{\circledR}\right)$ generation DES, Kobayashi group retrospectively evaluated 216 patients that had in-stent restenosis (ISR) and undergone repeat PCI under OCT or OFDI guidance. The authors found that compared with second-generation DES, the proliferation of neoatherosclerosis in terms of its length and arc was significantly greater and the fibrous cap was significantly thinner in first-generation DES, suggesting a more stable proliferation and stability of neoatherosclerosis on second-generation DES [16].

Despite the rate of stent thrombosis (ST) being low at around $1.83 \%$, it is such a catastrophic event that deserves attention. Nato et al. investigated the OCT and IVUS findings at 1 year follow-up, of 34 patients that had late and very-late ST treatment guided by intravascular imaging. The patients were divided at baseline according to the most probable causative factor of ST, such as malapposition (17 patients) or other findings (17 patients) and then re-assed at 1 year follow-up. They found that patients that presented with malapposition are more prone to have a worse healing pattern than those who presented with other causes, thus being at higher risk for recurrent ST [17].

\section{Evaluation of plaque and tissue characteristics}

Highlighting the importance of high resolution intravascular imaging on the decision making process, Gupta and colleagues presented a case of ambiguous angiogram imaging, that could lead to different treatments. By utilizing OCT and finding a honeycomb like structure, they were able to differentiate the thrombus recanalization from spontaneous dissection, ultimately delivering proper and directed treatment, avoiding unnecessary risks for the patient [18].

By utilizing other imaging modalities in conjunction with intravascular imaging, Kaichi et al. showed that this approach might give important insights to elucidate the pathophysiology of eruption of coronary atheroma in vivo in a case report of a patient presenting with ST elevation in inferior leads. With the help of IVUS and computer tomography they were able to conclude that the lipidic material from a plaque rupture, traveled into coronary circulation, resulting in a zero lipid core burden at the lesion site [19].

Lee et al. investigated whether aspirin have a protective effect on patients with vasospasm-induced coronary syndrome (VACS), by utilizing OCT to define this entity. In a retrospective cohort study they followed for 48 months 154 patients who presented with VACS: using propensity score matching those patients were divided in equal groups of nonaspirin and aspirin use. Myocardial infarction (17\% vs. 3\%, $\mathrm{p}=0.003)$ and chest pain recurrence ( $26 \%$ vs. $9 \%, \mathrm{p}=0.006)$ occurred more frequently in the non-aspirin group, showing that aspirin may have a protective effect on patients presenting with VACS [20].

\section{Endothelial shear stress and vessel physiology}

On an effort to understand the impact of vessel morphology on physiology, Pyxaras et al. combined the data of a motorized FFR pullback with an OCT pullback, thus being able to associate regions on the OCT with pressure variations on the FFR. With this approach they were able to show, on a 26 patients series, that sub-optimal FFR (defined as an FFR post-PCI <0.94) was associated with higher mean neointimal area (respectively: $1.06 \pm 0.80$ vs. $0.51 \pm 0.23 \mathrm{~mm}^{2}$; $\mathrm{p}=0.018$ ) and higher neointimal thickness of covered struts (respectively $0.11 \pm 0.07$ vs. $0.06 \pm 0.01 \mathrm{~mm} ; \mathrm{p}=0.021$ ), compared to patients with optimal FFR post-PCI. The study suggest that regions with sub-optimal FFR at baseline behave poorly and might be associated with worse outcomes [21].

Gutiérrez-Chico published an editorial highlighting the work done by $\mathrm{Wu}$ et al. on the field of shear stress and vessel biomechanics. By utilizing a simple coronary angiogram the authors were able to calculate the superficial wall stress, combining several motions of the vessel (such as radial stress, bending, stretching and twisting) and with the potential to be translated to a real world, live procedure use to guide treatment [22]. 


\section{Atherectomy and other novel treatments}

On the topic of atherectomy and plaque modification on peripheral vessels, Kuku et al. on behalf of the CELLO study investigators, showed that excimer laser is a promising approach in preventing limb amputation over 1-year follow-up. One of the main factors believed to cause restenosis is vessel wall injury during the baseline procedure. The authors described adventitial layer disruption in $<1 \%$ of the 1867 post ablation frames assessed by IVUS, which resulted in a secondary patency of $100 \%$ at 6 and 12 months, despite a lower percentage of primary patency. Those are very promising results and results from RCT are welcomed [23].

Oba and his groups described a case in which they performed an angioplasty using paclitaxel-coated balloon (SeQuent® Please; B. Braun, Melsungen, Germany) to treat an ISR due to a stent fracture observed by OFDI. They preferred this approach in order to avoid an possible increased inflammatory response by the stent fracture plus the new struts. They reported that 8 months later OFDI showed the total separation of the struts due stent fracture, coronary angiography revealed a satisfactory status of the lesion and the fibrous cap thickness was increased [24].

Rotational atherectomy (RA) for calcified coronary lesions is currently a hot topic and The International Journal of Cardiovascular Imaging covered two different publications on the theme. Kim et al. investigated using IVUS the effects of rotational atherectomy (Rotablator ${ }^{\mathrm{TM}}$ Rotational Atherectomy System) in calcified coronary lesions on a series of 38 patients. By utilizing this device with the rotational speed raging between 160,000 and 200,000 rpm they showed that minimum lumen area, maximum reverberation number, angle, and length increased post atherectomy, which indicates calcium modification. In addition newly visible perivascular tissue through previously solid calcium (indicating calcium fracture) was observed [25].

Still on rotational atherectomy, but this time utilizing OFDI and Rotalink Plus ${ }^{\mathrm{TM}}$ Rotational Atherectomy System (Boston Scientific Corporation, Natic, MA, USA) to investigate findings that predict good stent expansion post debulking, Kobayashi et al. performed a prospective, single center study, in which included 44 patients or 50 de novo severely calcified coronary lesions. His group found that the minimum thickness of calcium in the intima after RA was the most important calcium parameter associated with stent expansion. Second, stent expansion was significantly better in the lesions with dissection after RA, compared to lesions without dissection. Third, the minimum thickness of calcium in the intima and dissection formation after RA were independent predictors of good stent expansion [26].

\section{Side branches and bifurcation lesions}

In a review paper, Shrestha et al. highlighted the enormous importance that OCT have in assessing vulnerble plaque in coronary bifurcation lesions. Due to its spatial resolution of $10 \mu \mathrm{m}$ and faster pullback speed reducing artifacts formation, OCT can identify fibrous, fibrocalcific, lipid-rich coronary plaque and also measure the thickness of the fibrous cap, it directly visualizes micro-vessels within the coronary atherosclerotic plaque. With that information and its ability to provide lumen dimensions, vulnerable plaque's composition and characteristics, the author believes its clinical significance and several other essential findings associated with bifurcation lesions will guide bifurcation lesion intervention to a better outcome [27].

Chen et al. investigated whether IVUS guided treatment is better than angiography alone in treating unstable angina patients and Medina 1,1,1 or $0,1,1$ coronary bifurcation lesions. Over a 6-year period they included 310 patients in the IVUS guidance and utilized propensity matching score to pair with 620 patients in the angiography group. They found that MACE occurred in $10.0 \%$ of patients at 1-year followup and $15.2 \%$ at the 7 -year follow-up in the IVUS group, significantly different from $15.0 \%(\mathrm{p}=0.036)$ and $22.4 \%$ $(\mathrm{p}=0.01)$ in the angiography group, respectively. Compared to angiography guidance, IVUS guidance also resulted in a lower 7 -year cardiac death rate $(6.5$ vs. $1.3 \%, \mathrm{p}=0.002)$ and MI (8.4 vs. $2.3 \%, p<0.001)$, suggesting that intravascular imaging is crucial in obtaining good results when treating bifurcation lesions [28].

\section{Nuclear cardiology}

In 2018 different excellent paper in the field of nuclear cardiology were published in the journal. In this review we selected a few papers on the topics of new technological developments, myocardial flow reserve assessment and myocardial viability.

\section{New technological developments}

There is ongoing debate in the context of myocardial perfusion imaging (MPI) with CMR on the merits of using technically complex acquisition methods to achieve wholeheart spatial coverage, rather than conventional three-slice acquisition. As an adequately powered comparative study is difficult to achieve for two separate CMR Studies, Shariff et al. [29] compared whole-heart versus simulated threeslice coverage in a large existing SPECT data set from 651 patients with suspected CAD who underwent invasive angiography. Diagnostic accuracy for the three-slice and whole-heart approaches were similar (AUC 0.743 vs. 0.855 
respectively, $\mathrm{p}=0.07)$. However, the majority $(54 \%)$ of cases missed by three-slice imaging had primarily apical ischemia. Therefore, the authors conclude that caution is required when comparing the ischemic burden between three-slice and whole-heart datasets and that there is a need to establish prognostic thresholds specific to each approach.

\section{Assessment of myocardial flow reserve}

It is unclear whether myocardial flow reserve (MFR) derived by dynamic perfusion SPECT using a novel D-SPECT camera reflects the severity of coronary atherosclerosis. Iguchi et al. [30] studied 40 patients who underwent both a myocardial dynamic perfusion SPECT study and invasive coronary angiography. All patients underwent a rest/ stress SPECT imaging protocol using Tc-99m-sestamibi and dynamic acquisition was performed. Stress and rest flow was evaluated and global and regional MFR was calculated. The severity of coronary atherosclerosis was evaluated using the Gensini score. The main findings were that global MFR derived by dynamic perfusion SPECT using D-SPECT reflects the severity of coronary atherosclerosis. Also, regional MFR was significantly lower in regions with $>90 \%$ stenotic lesions compared with regions with $<90 \%$ stenotic lesions. Based on these results, global and regional MFR derived by dynamic perfusion SPECT may reflect the global and regional severity of coronary atherosclerosis. The total number of patients was however limited and no invasive fractional flow reserve measurements were performed, so clearly further studies are needed on this topic.

Combined MFR by PET and CT coronary angiography (CTA) is a promising toot for assessment of CAD. Prior studies of MFR/CTA has been performed as side-by-side interpretation, not as volume rendered, full hybrid analysis, with fused MFR/CTA. Braad et al. [31] developed a method for full hybrid analysis of MFR/CTA and determined the diagnostic value of side-by-side versus full hybrid MFR/ CTA with 15-O-water PET. They studied 44 patients who underwent stress/rest 15-O-water PET/CTA with invasive coronary angiography as reference. MFR showed a high reproducibility within and between observers of different expirience. The full hybrid model was not superior to sideby-side interpretation of MFR/CTA but proved better than MFR alone at vessel level with regard to specificity, positive predictive value, and accuracy, suggesting that this full hybrid analysis of MFR/CTA may become a clinical relevant tool for the assessment of CAD.

\section{Imaging the myocardial ischemic cascade}

Non-invasive imaging plays a growing role in the diagnosis and management of ischemic heart disease from its earliest manifestations of endothelial dysfunction to myocardial infarction along the myocardial ischemic cascade. Experts representing the North American Society for Cardiovascular Imaging and the European Society of Cardiac Radiology have provided an extensive review of the current status of non-invasive imaging for ischemic heart disease, along with the role of imaging for guiding surgical planning of patients who may require coronary artery bypass grafting. Special attention is given to the cost-effectiveness of the different imaging modalities in this excellent state-of-the art overview [32].

\section{Myocardial viability}

Positron emission tomography/computed tomography (PET/ CT) imaging with (18) F-radiolabeled fluorodeoxyglucose (FDG) is a powerful imaging technique for the assessment of myocardial viability in patient with CAD. Data on the role of cardiac viability imaging in elderly patients are however scarce. Namdar et al. [33] evaluated 89 patients $>65$ years (mean age 74 years) with CAD and LVEF $<40 \%$ with cardiac FDG PET/CT. Patients were stratified according to the presence/absence of viable myocardium and subsequent revascularization and followed for changes in LVEF and hard cardiovascular end-points. An increase in LVEF was only noted in patients with viable myocardium who underwent revascularization $(n=37$, increase of LVEF of $3.8 \%$ after $2 \pm 1$ years). Importantly viable myocardium was associated with better clinical outcomes in these elderly patients when revascularized. These findings are in line with many previous studies in similar younger patients and support the assessment of viability with FDG PET/CT in elderly patients with CAD and reduced LVEF.

Wang et al. [34] evaluated the prognostic value of LV viability and remodeling parameters in 126 patients with LV aneurysms. Patients underwent gated PET, gated SPECT and CMR and were followed for 3.9 years. Patients were divided into three groups by aneurysmal viability (mismatch score of aneurysm $\geq 2$ ) and $\mathrm{LV}$ remodeling (ESVI $>60 \mathrm{ml} / \mathrm{m}^{2}$ by gated PET): group 1 (viability -, LV remodeling -), group 2 (viability -, LV remodeling + ) and group 3 (viability,$+ \mathrm{LV}$ remodeling \pm ). $L V$ remodeling was associated with cardiac death during follow-up and only for patients in group 3 coronary revascularization was associated with better long-term survival. These data suggest that when using LV viability as a parameter for decision making regarding revascularization, also LV remodeling should be taken into account in CAD patients with $\mathrm{LV}$ aneurysm.

\section{Echocardiography}

In 2018 several interesting echocardiography studies appeared in the International Journal of Cardiovascular Imaging. Some of them are highlighted here. 


\section{Improving pulmonary hypertension diagnosis by using multiple transthoracic echocardiography views}

Pulmonary hypertension is a disease with severe morbidity and mortality. Echocardiography plays an essential role in the screening of pulmonary hypertension. The quality of the acquired continuous wave Doppler signal is the major limitation of the method and can greatly affect the accuracy of estimated pulmonary pressures. The aim of the study by Schneider et al. was to evaluate the clinical need to image from multiple ultrasound windows in patients with suspected pulmonary hypertension [35]. They prospectively evaluated 65 patients (43\% male, mean age 67.2 years) with echocardiography and right heart catheterization; $17 \%$ had invasively normal pulmonary pressures, $83 \%$ had pulmonary hypertension. Peak tricuspid regurgitation velocity was imaged in five echocardiographic views. Sufficient Doppler signal was recorded in $94 \%$ of the patients. Correlation for overall peak tricuspid regurgitation velocity with invasively measured systolic pulmonary artery pressure was $r=0.83(p<0.001)$. Considering all five imaging windows resulted in a sensitivity of $87 \%$, and a specificity of $91 \%$ for correct diagnosis of pulmonary hypertension with an AUC of 0.89 , which was significantly better as compared to sole imaging from the right ventricular modified apical four-chamber view (AUC $0.85, \mathrm{p}=0.0395$ ). Additional imaging from atypical views changed the overall peak tricuspid regurgitation velocity in $32 \%$ of the patients. A multiple-view approach changed the echocardiographic diagnosis of pulmonary hypertension in $11 \%$ of the patients as opposed to sole imaging from an apical four-chamber view.

\section{Does pocket-size ultrasound imaging improve the accuracy of the initial assessment of suspected pulmonary embolism?}

Pulmonary embolism onset is frequently neglected due to the non-specific character of its symptoms. Pocket-size imaging devices present an opportunity to implement imaging diagnostics into conventional physical examination. The aim of the study by Filipiak-Strzecka et al. [36] was to test the hypothesis that supplementation of the initial bedside assessment of patients with suspected pulmonary embolism with four-point compression venous ultrasonography and right ventricular size assessment with the use of pocket-size ultrasound imaging equipped with dual probe could positively influence the accuracy of clinical predictions. A single-centre, prospective analysis was conducted on 100 patients (47 men, mean age $68 \pm 13$ years) with suspected pulmonary embolism. Clinical assessment on the basis of Wells and revised Geneva score and physical examination were supplemented with four-point compression venous ultrasonography and right ventricular size assessment with the use of pocketsize ultrasound imaging. The mean time of pocket size scanning was $4.9 \pm 0.8 \mathrm{~min}$ and was universally accepted by the patients. Fifteen patients had deep venous thrombosis and right ventricular enlargement was observed in 59 patients. Pulmonary embolism was confirmed in 24 patients. If both the venous ultrasonography was positive and right ventricle enlarged, the specificity was $100 \%$ and sensitivity $54 \%$, ROC AUC 0.771 (95\% CI 0.68-0.85). The Wells rule within the study population had a specificity of $86 \%$ and sensitivity of 67\%, ROC AUC 0.776 (95\% CI 0.681-0.853, p<0.0001). Similar values calculated for the revised Geneva score were as follows: specificity $58 \%$ and sensitivity $63 \%$, ROC AUC 0.664 (95\% CI 0.563-0.756, $\mathrm{p}=0.0104$ ). Supplementing the revised Geneva score with additional criteria of venous ultrasonography result and right ventricular measurement resulted in significant improvement of diagnostic accuracy. The difference between ROC AUCs was $0.199(95 \% \mathrm{Cl}$ $0.0893-0.308, \mathrm{p}=0.0004)$. Similar modification of Wells score increased ROC AUC by 0.133 (95\% CI $0.0443-0.223$, $\mathrm{p}=0.0034$ ). Despite the well-acknowledged role of the pulmonary embolism clinical risk assessment scores the diagnostic process may benefit from the addition of basic bedside ultrasonographic techniques.

\section{Does transducer frequency setting affect speckle tracking measures?}

Speckle tracking echocardiography is an emerging technique, which is currently being included in clinical guidelines. Olsen et al. sought to investigate the impact of transducer frequency settings on speckle tracking derived measures [37]. The study comprised of 22 subjects prospectively enrolled for a randomized controlled trial (LOOPstudy). Patients were above 70 years of age with increased risk of stroke, and had an echocardiogram performed, which included focused images of the left ventricle. Focused images were obtained with the transducer frequency set at both 1.7/3.3 and 1.5/3.0 MHz. The images were obtained immediately after each other at the exact same position for the two settings. Speckle tracking was performed in three apical projections, allowing for acquisition of layered global longitudinal strain and strain rate measures. Concordance between the frequency settings was tested for endo-, mid-, and epicardial global longitudinal and strain rates by coefficients of variation, bias coefficients and visually displayed by Bland-Altman plots. Bland-Altman plots did not reveal any significant over- or underestimation of any speckle tracking measure. Bias coefficients showed that none of the measurements differed significantly between the two settings. Changing between transducer frequency settings does not systematically derange speckle tracking measures. The authors concluded that one can safely reduce the transducer 
frequency without compromising the validity of speckle tracking derived measures.

\section{Standardizing strain measurements of the right ventricle}

Right ventricular strain values by two-dimensional strain echocardiography can be used as objective markers of right ventricular systolic function. However, there is little data about normal reference right ventricular strain values according to age and gender. Park et al. analysed right ventricular strain data from the digitally stored echocardiographic images from NORMAL (Normal echOcardiogRaphic diMensions and functions in KoreAn popuLation) study for the measurement of normal echocardiographic values performed in 23 Korean university hospitals [38]. They enrolled in total 1003 healthy persons in the NORMAL study. Of them, they analysed two-dimensional right ventricular strain values in 493 subjects ( 261 females, mean $47 \pm 15$ years old) only with echocardiographic images by GE machines. Their LV systolic and diastolic functions were normal. Total right ventricular global longitudinal peak systolic strain was $-21.5 \pm 3.2 \%$. Females had higher absolute values than males. Younger ( $<50$ years old) females had higher absolute values than age matched males. Total right ventricular global longitudinal peak systolic strain in females gradually increased according to age ( $p$ for trend $=0.002$ ) and becomes almost similar in age $\geq 50$ years. However, this trend was not seen in males ( $p$ for trend $=0.287$ ), and younger males had similar values to that of older men.

\section{Is right atrial strain associated with right atrial pressure?}

Echocardiographic assessment of right atrial pressure from inferior vena cava dimension may underestimate catheterderived right atrial pressure. As right atrial deformation, measured by speckle tracking, is preload-dependent, Wright et al. hypothesized that right atrial strain may improve estimation of catheter derived right atrial pressure [39]. Right atrial strain components were measured in 125 of 175 patients who had echocardiography and invasive measures of right atrial pressure. (median difference 1 day). To determine whether right atrial strain measures differentiated patients with correct vs. incorrect right atrial pressure from inferior vena cava assessment, categories with values $<3$, 8 and $>15 \mathrm{mmHg}$ were compared with groups $<3,4-7$, $8-10,11-14$ and $>15 \mathrm{mmHg}$. Non-invasively determined right atrial pressure was significantly lower $(\mathrm{p}=0.001)$ than invasively determined right atrial pressure, with a weak correlation $(r=0.35, p<0.001)$. Right atrial strain components were associated with right atrial size, right ventricular function and inferior vena cava size. In those with inferior vena cava derived right atrial pressure $>15 \mathrm{mmHg}$, half of patients were categorized into right atrial pressure $<10 \mathrm{mmHg}$. There were no significant differences in right atrial characteristics that differentiated patients in whom echocardiographic estimation of right atrial pressure was inaccurate. Right atrial strain measures were feasible, and had associations with right atrial size, right ventricular systolic function and inferior vena cava size. Right atrial strain was significantly different between those with normal vs. raised pressure, but it did not identify those with incorrect echocardiographic assessment of right atrial pressure.

\section{Can left atrial deformation parameters predict for paroxysmal atrial fibrillation in patients with end-stage renal disease?}

It is widely known that various factors contribute to left atrial mechanical dysfunction in patients with end stage renal disease. However, the connection between atrial dysfunction and arrhythmic events such as paroxysmal atrial fibrillation, in this group of patients, remains unclear. Papadopoulos et al. prospectively evaluated the association between left atrial deformation indices and paroxysmal atrial fibrillation in 79 patients with end stage renal disease and preserved left ventricular systolic function [40]. All patients underwent a baseline comprehensive echocardiography study and were followed for a mean period of $16 \pm 5$ months. Paroxysmal atrial fibrillation episodes, first and the following events, were reported. Left atrial longitudinal strain reflecting left atrial reservoir function and left atrial longitudinal strain rate reflecting left atrial pump function were specifically evaluated as left atrial deformation indices of interest, using 2D speckle tracking echocardiography. At the end of follow up period nine patients died. Fifteen of the rest 70 reported one or more episodes of paroxysmal atrial fibrillation. Left atrial indexed volumes were significantly higher in patients with paroxysmal atrial fibrillation $(32 \pm 26$ vs. $21.5 \pm 9 \mathrm{ml} / \mathrm{m}^{2}, \mathrm{p}=0.002$ ), mean left atrial strain was significantly reduced $(17 \pm 7$ vs. $27 \pm 9 \%, \mathrm{p}<0.001)$ as well as mean left atrial strain rate $(-1.19 \pm 0.5$ vs. $-1.95 \pm 0.5$ $1 / \mathrm{s}, \mathrm{p}<0.001)$. Multivariate analysis showed that left atrial strain rate when adjusted with age together with paroxysmal atrial fibrillation history remained the single most significant echocardiographic parameter for paroxysmal atrial fibrillation prediction. Further prospective studies are needed to validate its relevance in routine clinical practice.

\section{Right ventricular function and mechanics in chemotherapy- and radiotherapy-naïve cancer patients}

Tadic et al. evaluated right ventricular structure, function and mechanics in cancer patients before initiation of 
chemo- or radiotherapy, and the association between cancer and decreased right ventricular longitudinal strain [41]. This retrospective investigation included 101 chemo- and radiotherapy-naïve patients with solid cancer and 38 age- and gender-matched controls with similar cardiovascular risk profile. Echocardiographic examination and strain evaluation was performed in all participants. Right ventricular structure and right ventricular systolic and diastolic function estimated with conventional echocardiographic parameters were similar between the cancer patients and controls. However, right ventricular global longitudinal strain was significantly decreased in the cancer patients than in controls. The same was revealed for right ventricular free wall endocardial and mid-myocardial longitudinal right ventricular strains, whereas difference was not found in right ventricular free wall epicardial longitudinal strain. The presence of cancer was independently of age, gender, body mass index, left ventricular hypertrophy, diabetes, hypertension and pulmonary pressure associated with reduced right ventricular global longitudinal strain, as well as with decreased free wall right ventricular longitudinal strain. Right ventricular strain is deteriorated in the chemo- and radiotherapy-naïve cancer patients.

\section{Echocardiographic features of PFOs and paradoxical embolism: a complicated puzzle}

Finally we would like to recommend a review on patent foramen ovale and stroke [42]. Patent foramen ovale (PFO) is a residual, oblique, slit or tunnel like communication in the atrial septum that persists into adulthood. It is usually an incidental finding with no clinical repercussions. Nevertheless, recent evidence supports the association between the presence of a patent foramen ovale and a number of clinical conditions, most notably cryptogenic stroke. There is enough evidence that paradoxical embolism is a mechanism which can explain this association. Patient characteristics and certain echocardiography-derived anatomical and hemodynamic features of patent foramen ovale provide great assistance in estimating the probability of paradoxical embolism. In this review, the authors describe patent foramen ovale embryology and anatomy. They extensively present the available data on clinical, anatomical and hemodynamic features of PFOs which have been correlated with increased likelihood of paradoxical embolism and recent evidence of therapeutic management.

\section{Magnetic resonance imaging}

There were a number of important contributions in cardiovascular MRI (CMR) in 2018. Many of these were related to the use of T1 and T2 mapping methods. Piechnik and
Jerosch-Herold reviewed physical and physiological mechanisms which underlie the interpretation of T1 measurements and extracellular volume fraction (ECV) [43]. The variability of native myocardial $\mathrm{T} 1$ values was assessed by Lin et al. [44]. An automated regional analysis of myocardial native $\mathrm{T} 1$ of the left ventricle was presented [45]. Lagan et al. reviewed the application of multi-parametric CMR in myocarditis and systemic inflammatory diseases [46]. While normal left ventricular function was found in patients who have undergone orthotopic heart transplantation, these patients have higher ECV and intracellular lifetime of water (a measure of cardiomyocyte hypertrophy compared with age-matched volunteers [47]). In related work, Yuan et al. found increased ECV and T2 values in asymptomatic heart transplant patients [48]. The inversion time used for late gadolinium enhancement was found to be non-inferior to ECV for distinguishing cardiac amyloidosis from hypertrophic cardiomyopathy [49]. T1 mapping and rest-stress T1 mapping of the spleen demonstrated that the splenic switch-off sign is not a reliable indicator of stress adequacy during adenosine perfusion CMR [50]. A T1-mapping based synthetic inversion recovery approach determine the optimal inversion time for LGE was presented [51]. Right ventricular T1 mapping and ventricular dysfunction were found to be correlated by Jellis et al. [52]. Podlesnikar et al. reviewed the potential for CMR to assess myocardial fibrosis in valvular heart disease [53].

There also were important contributions in myocardial strain assessment with CMR. A strong association was found for 3D myocardial strain and T1 mapping in patients with cardiac amyloidosis [54]. Other workers found decreased global longitudinal strain and strain rate in the epicardial and endocardial layers in patients with cardiac amyloidosis secondary to multiple myeloma [55]. Left ventricular circumferential strain was assessed by CMR tagging, CMR feature tracking and speckle tracking echocardiography in patients being evaluated for cardiac resynchronization therapy showing better agreement for both CMR methods compared with echocardiography [56]. Both myocardial perfusion reserve and global longitudinal strain were found to be abnormal in patients with orthotopic heart transplantation but not related to allograft rejection, myocardial scar/fibrosis or allograft dysfunction [57]. Reference ranges for 3D feature tracking were provided [58]. Strain values assessed with CMR feature tracking were found to have an association with age and gender but not other cardiovascular risk factors in asymptomatic Asian subjects [59].

A fully-automated method for left ventricular mass and volume analysis was applied to the UK Biobank population and found to have good agreement to manual analysis [60]. The peak filling and emptying rates of both ventricles were found to be highly reproducible in patients with pulmonary arterial hypertension [61]. Chronic aortic regurgitation 
patients taking metroprolol were found to have significantly lower mean heart rate, cardiac power index and rate-pressure product compared with those taking losartan however the aortic regurgitation fraction was greater [62]. Passive left atrial ejection fraction was found to be the most sensitive of various functional indices for function and structure in a CMR assessment patients with type 2 diabetes mellitus compared with healthy controls [63].

The role of CMR for imaging ischemic heart disease was reviewed [64, 65]. Incomplete coverage of the left ventricle using three short axis slices was not found adversely affect diagnostic accuracy compared with a whole heart assessment but may fail to detect apical ischemia and underestimate the extent and severity of perfusion defects [66]. Whole heart CMR perfusion was fused with coronary CTA permitting correlation with culprit coronary lesions [67].

The utility of CMR in patients with hypertrophic cardiomyopathy (HCM) was demonstrated in several publications. Multiparametric CMR was used to distinguish HCM from other causes of left ventricular hypertrophy [68]. Maragiannis et al. found significantly longer $\mathrm{T} 1$ relaxation time in patients with HCM compared with controls which was associated with left ventricular diastolic dysfunction [69]. Gommans et al. found high T2-weighted signal intensity was associated with higher risk of sudden death in patients with HCM [70]. Left atrial indexed volume and LGE were found to be predictors of poor clinical outcomes in patients with end-stage HCM [71].

Diffusion-weighted CMR was found to be comparable to PET-CT in the follow-up of patients with chronic periaortitis [72]. The use of a blood pool contrast agent for gated thoracic MRA at $3 \mathrm{~T}$ was found to improve the image quality compared with a non-contrast technique and provided more reproducible measurements of the aortic annulus area [73].

There were several publications highlighting the use phase contrast in the aorta. Kamphuis et al. found good scanrescan reproducibility of diastolic left ventricular kinetic energy, viscous energy loss, and vorticity assessment [74]. Ferumoxytol was found to be a good alternative to gadofosveset trisodium for 4D flow image quality [75]. Pulse wave velocity calculated from $4 \mathrm{D}$ flow of the aorta at $3 \mathrm{~T}$ and was found to significantly correlated to measures of arterial stiffness determined with transesophageal echocardiography in stroke patients [76]. Forward displacement in the ascending aorta was suggested as a surrogate marker for wall shear stress in patients with aortic regurgitation [77]. The aortic stiffness of ascending aorta was found to be increased in children having coarctation of the aorta repaired surgically or balloon angioplasty whereas no difference was observed with those treated with a stent compared with controls [78].

In other work related to congenital heart disease, the effects of breath holding for quantification of left-to-right shunt in patients with secundum atrial defect were and found to be best with expiratory breath hold as compared with either free breathing or inspiratory breath hold using invasive oximetry as the reference standard [79]. Diffuse fibrosis was found to be common in the left ventricle but not right ventricle in patients with transposition of the great arteries late after atrial switch repair [80]. The Grothoff approach to assess left ventricular non-compaction was found to better capture the extent of non-compacted tissue compare with the Jacquier approach [81].

The use of CMR for atrial tissue characterization in arrhythmias and guidance for electrophysiology procedure guidance was reviewed [82]. Time-resolved contrastenhanced MRA was found to have superior image quality compared to traditional contrast-enhanced MRA in patients with atrial fibrillation being evaluated for ablation procedures [83].

The feasibility of CMR guidance for endomyocardial biopsy was demonstrated in an in vivo porcine model [84]. The same group also demonstrated the feasibility of MRI guided renal denervation using active tracking in a porcine model [84].

\section{Computed tomography}

In 2018 a wide variety of articles describing clinical applications of cardiovascular CT have been published in the journal. In the following review, we describe a few highlights grouped by clinical topics:

\section{Coronary imaging, myocardial perfusion, CT-FFR, and coronary calcium scoring}

Coronary CTA is well established, but remains limited in the assessment of clinical significance of intermediate and calcified lesions. Non-invasive assessment of lesion significance with rest/stress myocardial perfusion imaging and fractional flow reserve (FFRCT) derived from coronary computed tomography angiography therefore are relevant topics.

Ghekiere et al. examined 37 patients with intermediate stenoses $(n=39)$ identified with coronary CTA [85]. The correlations between FFRCT and adenosine perfusion cardiac magnetic resonance (CMR) with invasive FFR were retrospectively evaluated. Invasive FFR values correlated equally strongly with FFRCT $(r=0.675 ; p<0.001)$ and the relative CMR myocardial perfusion index $(r=-0.63)$ $(p<0.001 ; z=6.72)$ for assessment of lesion-specific ischemia.

CT perfusion and FFRCT have their own limitations, which were evaluated in other papers:

In an ex vivo model, van Assen et al. evaluated the effect of temporal sampling rate in dynamic CT myocardial perfusion imaging (CTMPI) on myocardial blood flow (MBF) in 
three porcine hearts using different acquisition modes [86]. True MBF was calculated using input flow and heart weight. Significant differences in MBF between shuttle, non-shuttle and continuous mode were found. The median MBF in the shuttle mode was $56 \%$ lower than the true MBF. In non-shuttle and continuous mode, the underestimation was $41 \%$ and $18 \%$. The authors conclude, that limited temporal sampling rate in standard dynamic CTMPI techniques contributes to substantial underestimation of true MBF.

Nozue et al. examined the predictors that results in cases being inappropriate for FFRCT processing [87]. From a group of 50 patients, FFRCT could not be derived in 11 (22\%). Heart rate at CCTA examination (72 beats/min vs. 63 beats/min; $p=0.007$ ) and Agatston score (665 vs. 33; $\mathrm{p}=0.002$ ) were significantly higher in these patients. Multivariate logistic regression analyses revealed that heart rate at CCTA examination [OR 1.348 (95\% CI 1.167-1.556); $\mathrm{p}<0.001$ ] and Agatston score [OR $1.002(95 \%$ CI 1.000-1.003]; $p=0.004$ ) were significant, independent factors associated with non-measurability of FFRCT. The frequency of poor image quality was highest in patients with heart rate $>65$ beats/min and Agatston score $>400$ $(\mathrm{p}<0.0001)$.

Interestingly, two papers from van der Werf et al. about coronary calcium scoring discussed that there is still a need to standardize acquisition in particular when comparing results across vendor platforms [88, 89]. The studies examined the influence of heart rate on coronary calcium scores (CCS) using a dynamic phantom as the influence of iterative reconstruction on coronary calcium scores (CCS) at different heart rates for four state-of-the-art CT systems from different manufacturers.

\section{Structural and valvular heart disease}

Beyond TAVR, image guidance is important for an increasing number of structural and valvular heart disease interventions.

Lindner et al. examined CT imaging in the context of left atrial appendage closure (LAAC) [90]. The authors applied a standardized imaging protocol to detect and quantify peri-device leaks (PDL) after LAAC. CTA datasets of 49 consecutive patients 6 months after successful LAAC were acquired on a third generation dual-source computed tomography system. Overall PDL rate was 31\%. Leak rates among different left atrial appendage morphologies varied largely. Windsock type had the highest incidence of PDL (47\%). AMPLATZER $^{\text {TM }}$ AMULET $^{\text {TM }}$ device type revealed slightly higher PDL rates than WATCHMANTM type and showed larger leaks. However, no statistical differences were found.

Obasare et al. examined the role of three-dimensional (3D) printing of the LAA in 14 patients who underwent Watchman device (WD) implantation [91]. The patients underwent cardiac computed tomography (CCT) with $3 \mathrm{D}$ printing to produce a latex model of the LAA for preprocedure planning. The model correlated perfectly with implanted device size $\left(\mathrm{R}^{2}=1 ; \mathrm{p}<0.001\right)$, while TEE-predicted size showed inferior correlation $\left(\mathrm{R}^{2}=0.34 ; 95 \% \mathrm{CI}\right.$ $0.23-0.98, p=0.03$ ). Use of the model was associated with reduced procedure time ( $70 \pm 20$ vs. $107 \pm 53 \mathrm{~min}, \mathrm{p}=0.03)$, anesthesia time ( $134 \pm 31$ vs. $182 \pm 61 \mathrm{~min}, \mathrm{p}=0.03)$, and fluoroscopy time ( $11 \pm 4$ vs. $20 \pm 13 \mathrm{~min}, \mathrm{p}=0.02)$. Absence of peri-device leak was also more likely when the model was used ( 92 vs. $56 \%, \mathrm{p}=0.04$ ).

3D and 4D CT imaging is also applied to the assessment of patient with endocarditis (IE).

Sims et al. compared cardiac CTA and TEE in the detection of endocarditic lesions [92]. 255 adults who underwent surgery for IE at the Mayo Clinic Rochester between January 1, 2006 and June 1, 2014 were identified retrospectively. 251 patients underwent TEE and 34 patients underwent cardiac CTA. TEE had statistically higher detection of vegetations ( 95.6 vs. $70.0 \%, \mathrm{p}<0.0001)$ and leaflet perforations ( 81.3 vs. $42.9 \%, \mathrm{p}=0.02$ ) as compared to cardiac CTA. For detection of abscess/pseudoaneurysm TEE had a similar sensitivity to cardiac CTA ( 90.5 vs. $78.4 \%, p=0.21$ ).

\section{Congenital heart disease and ACHD}

Goo et al. examined coronary artery anomalies on preoperative cardiac CT 318 children with tetralogy of Fallot or Fallot type of double outlet right ventricle were reviewed and compared with surgical findings [93]. Incidences of total and surgically critical coronary artery anomalies, concordance rate between cardiac $\mathrm{CT}$ and surgical findings, and diagnostic accuracy of cardiac CT were assessed. In addition, the types of surgical modifications for surgically critical coronary artery anomalies were reviewed. The incidences of total and surgically critical coronary artery anomalies were $8.5 \%$ (27/318) and 5.0\% (16/318), respectively. The concordance rate between cardiac $\mathrm{CT}$ and surgical findings was $95.0 \%$ (302/318). The diagnostic accuracy of cardiac CT was $96.9 \%$ (308/318).

Xiang et al. examined 70 patients with total anomalous pulmonary venous connection (TAPVC) between May 2014 and June 2017 in Hunan Children's Hospital [94]. Clinical data and computed tomography imaging were reviewed, and survival time was recorded. Pulmonary venous obstruction (PVO) was found in 30 (42.9\%) of 70 patients in this group. Of all concurrent abnormalities, atrial septal defect (ASD) was the most common (98.6\%), followed by patent ductus arteriosus (PDA; 31, 44.3\%), and persistent left superior vena cava (PLSVC; 5, 7.1\%). 1, 3, 6 and 12-month survival rates were $76,61,49$, and $38 \%$ respectively. Risk factors for mortality in multivariable analysis comprised PVO, McGoon index (MGI), and mode of delivery. 


\section{Software advances}

Advances in software, including machine learning and artificial intelligence applications are an exciting aspect of imaging.

Gao et al. assess the quality of a new tool for the semiautomatic quantification of thoracic aortic dimensions in 29 patient with two CTA scans for which the official clinical report indicated an increase in aortic diameters [95]. Aortic maximal cross-sectional diameters of baseline and follow-up studies generated semi-automatically by the software were compared with corresponding manual measurements. The semi-automatic measurements were performed at seven landmarks defined on the baseline scan by two operators. The semi-automatic software generated results in 27 patients (93.1\%), demonstrating excellent intraclass correlation coefficients (all values $\geq 0.91$ ) and small differences. The semiautomatic tool decreased the processing time by $40 \%$ (13 vs. $22 \mathrm{~min}$ ).

Pourmorteza et al. examined stretch quantification of endocardial engraved zones (SQUEEZ) as a measure of regional cardiac function in an animal model [96]. The authors determine the effect of lowering the radiation dose on the precision of automatic SQUEEZ assessments of RWMA. Chronic myocardial infarction was induced in ten swine and CT was performed 5-11 months post infarct using first-pass contrast enhanced segmented cardiac function scans on a 320-detector row scanner at $80 \mathrm{kVp} / 500 \mathrm{~mA}$. Images were reconstructed at end diastole and end systole with both filtered back projection and using the "standard" adaptive iterative dose reduction (AIDR) algorithm. For each acquisition, nine lower dose acquisitions were created. Regional wall motion abnormality could be quantified with good precision from low dose acquisitions, using SQUEEZ, as long as the blood-myocardium CNR stayed above 4 .

Abd Alamir et al. evaluated the effectiveness of using computer aided diagnosis in the triage of low to intermediate risk emergency chest pain patients with Coronary Computed Tomographic Angiography (CCTA) [97]. The authors compared the diagnostic capability of computer aided diagnosis to human readers in $923 \mathrm{ED}$ patients with chest pain and examined index and 30 days outcomes by diagnosis for computer analysis and the human reader. $60 \%$ of cases could be triaged by the computer. Sensitivity was approximately $85 \%$ for 64 and 320 slice scanners, with specificity at $50.6 \%$ for the 64 slice and at $56.5 \%$ for the 320 slice scanner (per person measures). The NPV was 97.8 and 97.1 for the 64 and 320 slice scanners, respectively. The ROC for Computer Aided Diagnosis for the 64 and 320 Slice Scanners, using the human reader as the gold standard was 0.6794 and 0.7097 respectively. The index and 30 day outcomes were consistent for the human reader and computer aided diagnosis interpretation.

\section{Compliance with Ethical Standards}

Conflict of Interest The authors declare that they have no conflict of interest.

\section{References}

1. Detter C, Russ D, Kersten JF et al (2018) Qualitative angiographic and quantitative myocardial perfusion assessment using fluorescent cardiac imaging during graded coronary artery bypass stenosis. Int J Cardiovasc Imaging 34:159. https://doi.org/10.1007/ s10554-017-1212-1

2. Hayıroğlu M, Keskin M, Uzun AO et al (2018) Predictive value of SYNTAX score II for clinical outcomes in cardiogenic shock underwent primary percutaneous coronary intervention; a pilot study. Int J Cardiovasc Imaging 34:329. https://doi.org/10.1007/ s10554-017-1241-9

3. Strisciuglio T, Di Gioia G, Chatzikyriakou S et al (2018) Left atrial volume computed by $3 \mathrm{D}$ rotational angiography best predicts atrial fibrillation recurrence after circumferential pulmonary vein isolation. Int J Cardiovasc Imaging 34:337. https://doi. org/10.1007/s10554-017-1243-7

4. Kunio M, O'Brien CC, Lopes AC et al (2018) Vessel centerline reconstruction from non-isocentric and non-orthogonal paired monoplane angiographic images. Int J Cardiovasc Imaging 34:673. https://doi.org/10.1007/s10554-017-1275-z

5. Karabağ Y, Çă̆daş M, Rencuzogullari I et al (2018) Comparison of SYNTAX score II efficacy with SYNTAX score and TIMI risk score for predicting in-hospital and long-term mortality in patients with ST segment elevation myocardial infarction.. Int J Cardiovasc Imaging 34:1165. https://doi.org/10.1007/s10554-018-1333-1

6. Hayıroğlu M, Çınar T, Bıçakçı B et al (2018) Predictors of femoral hematoma in patients undergoing elective coronary procedure: a trigonometric evaluation. Int J Cardiovasc Imaging 34:1177. https ://doi.org/10.1007/s10554-018-1339-8

7. Maccagni D, Benincasa S, Bellini B et al (2018) Noise reduction technology reduces radiation dose in chronic total occlusions percutaneous coronary intervention: a propensity scorematched analysis. Int J Cardiovasc Imaging 34:1185. https://doi. org/10.1007/s10554-018-1343-Z

8. Tian Y, Yuan Y, Lu H et al (2018) Analysis of anomalous origin of coronary arteries by coronary angiography in Chinese patients with coronary artery disease. Int J Cardiovasc Imaging 34:1331. https://doi.org/10.1007/s10554-018-1350-0

9. Her AY, Shin ES, Lee JM et al (2018) Paclitaxel-coated balloon treatment for functionally nonsignificant residual coronary lesions after balloon angioplasty. Int J Cardiovasc Imaging 34:1339. https ://doi.org/10.1007/s10554-018-1351-z

10. Hideo-Kajita A, Garcia-Garcia HM, Kuku KO et al (2018) Clinical outcomes of complete revascularization using either angiography-guided or fractional flow reserve-guided drug-eluting stent implantation in non-culprit vessels in ST elevation myocardial infarction patients: insights from a study based on a systematic review and meta-analysis. Int J Cardiovasc Imaging 34:1349. https ://doi.org/10.1007/s10554-018-1362-9

11. Sonaglioni A, Baravelli M, Vincenti A et al (2018) A new modified anthropometric haller index obtained without radiological exposure. Int J Cardiovasc Imaging 34:1505. https://doi. org/10.1007/s10554-018-1366-5

12. Kuku KO et al (2018) Optical coherence tomography-guided percutaneous coronary intervention compared with other imaging guidance: a meta-analysis. Int $\mathbf{J}$ Cardiovasc Imaging 34(4):503-513 
13. Lv X et al (2018) Healing score of the Xinsorb scaffold in the treatment of de novo lesions: 6-month imaging outcomes. Int $\mathrm{J}$ Cardiovasc Imaging 34(7):1009-1016

14. Gil RJ et al (2018) Comparative assessment of three drug eluting stents with different platforms but with the same biodegradable polymer and the drug based on quantitative coronary angiography and optical coherence tomography at 12-month follow-up. Int J Cardiovasc Imaging 34(3):353-365

15. Kobayashi $\mathrm{N}$ et al (2018) Very early neointimal coverage of new biodegradable polymer drug-eluting stent compared with durable polymer everolimus-eluting stent evaluated by optical frequency domain imaging. Int J Cardiovasc Imaging 34(4):515-522

16. Kobayashi $\mathrm{N}$ et al (2018) Differences between first-generation and second-generation drug-eluting stent regarding in-stent neoatherosclerosis characteristics: an optical coherence tomography analysis. Int J Cardiovasc Imaging 34(10):1521-1528

17. Nato $\mathrm{M}$ et al (2018) One-year optical coherence tomography findings in patients with late and very-late stent thrombosis treated with intravascular imaging guided percutaneous coronary intervention. Int J Cardiovasc Imaging 34(10):1511-1520

18. Gupta PK et al (2018) Honeycomb-like appearance on optical coherence tomography in right coronary artery. Int J Cardiovasc Imaging 34(3):343-344

19. Kaichi R, Kataoka Y, Yasuda S (2018) Erupted coronary atheroma: insights from multi-modality imaging. Int $\mathrm{J}$ Cardiovasc Imaging 34(10): 1669-1671

20. Lee Y, Park HC, Shin J (2018) Clinical efficacy of aspirin with identification of intimal morphology by optical coherence tomography in preventing event recurrence in patients with vasospasminduced acute coronary syndrome. Int J Cardiovasc Imaging 34(11):1697-1706

21. Pyxaras SA et al (2018) In-stent fractional flow reserve variations and related optical coherence tomography findings: the FFR-OCT co-registration study. Int J Cardiovasc Imaging 34(4):495-502

22. Gutierrez-Chico JL (2018) Superficial wall stress: the long awaited comprehensive biomechanical parameter to objectify and quantify our intuition. Int J Cardiovasc Imaging 34(6):863-865

23. Kuku KO et al (2018) Intravascular ultrasound assessment of the effect of laser energy on the arterial wall during the treatment of femoro-popliteal lesions: a CliRpath excimer laser system to enlarge lumen openings (CELLO) registry study. Int J Cardiovasc Imaging 34(3):345-352

24. Oba Y et al (2018) A drug-coated balloon effectively treated instent restenosis due to a stent fracture. Int J Cardiovasc Imaging 34(2):169-170

25. Kim SS et al (2018) Intravascular ultrasound assessment of the effects of rotational atherectomy in calcified coronary artery lesions. Int J Cardiovasc Imaging 34(9):1365-1371

26. Kobayashi $\mathrm{N}$ et al (2018) Optical frequency-domain imaging findings to predict good stent expansion after rotational atherectomy for severely calcified coronary lesions. Int J Cardiovasc Imaging 34(6):867-874

27. Shrestha $\mathrm{R}$ et al (2018) A review in enormity of OCT and its enduring understanding of vulnerable plaque in coronary bifurcation lesion. Int J Cardiovasc Imaging 34(11):1679-1684

28. Chen L et al (2018) Intravascular ultrasound-guided drug-eluting stent implantation is associated with improved clinical outcomes in patients with unstable angina and complex coronary artery true bifurcation lesions. Int J Cardiovasc Imaging 34(11):1685-1696

29. Shariff B, Motwani M, Arsanjani R et al (2018) Impact of incomplete ventricular coverage on diagnostic performance of myocardial perfusion imaging. Int J Cardiovasc Imag 34:661-669

30. Iguchi N, Utanohara Y, Suzuki Y et al (2018) Myocardial flow reserve derived by dynamic perfusion single-photon emission computed tomography reflects the severity of coronary atherosclerosis. Int J Cardiovasc Imag 34(9):1493-1501
31. Braad PE, Pedersen KT, Petersen H et al (2018) 15-O-water myocardial flow reserve PET and CT angiography by full hybrid PET/ $\mathrm{CT}$ as a potential alternative to invasive angiography. Int J Cardiovasc Imag 34:2011-2022

32. Oudkerk M, Bluemke D, de Boer M et al (2018) Imaging the myocardial ischemic cascade. Int J Cardiovasc Imag 34:1249-1263

33. Namdar M, Rager O, Priamo J et al (2018) Prognostic value of revascularising viable myocardiu in elder patients with stable coronary artery disease and left ventricular dysfunction: a PET/ CT Study. Int J Cardiovasc Imaging 34(10):1673-1678

34. Wang W, Li X, Tian C et al (2018) Cardiac death in patients with left ventricular aneurysm, remodeling and myocardial viability by gated TC-MIBI SPECT and gated FDG-PET. Int J Cardiovasc Imaging 34:485-493

35. Schneider M, Pistritto AM, Gerges C et al (2018) Multi-view approach for the diagnosis of pulmonary hypertension using transthoracic echocardiography. Int J Cardiovasc Imaging 34:695

36. Filipiak-Strzecka D, Kasprzak JD, Lipiec P (2018) Brief cardiovascular imaging with pocket-size ultrasound devices improves the accuracy of the initial assessment of suspected pulmonary embolism. Int J Cardiovasc Imaging 34:1595

37. Olsen FJ, Svendsen JH, Køber L et al (2018) Impact of transducer frequency setting on speckle tracking measures. Int J Cardiovasc Imaging 34:457

38. Park JH, Choi JO, Park SW et al (2018) Normal references of right ventricular strain values by two-dimensional strain echocardiography according to the age and gender. Int J Cardiovasc Imaging 34:177

39. Wright LM, Dwyer N, Wahi S et al (2018) Association with right atrial strain with right atrial pressure: an invasive validation study. Int J Cardiovasc Imaging 34:1541

40. Papadopoulos CE, Pagourelias E, Bakogiannis C et al (2018) Left atrial deformation as a potent predictor for paroxysmal atrial fibrillation in patients with end-stage renal disease. Int J Cardiovasc Imaging 34:1393

41. Tadic M, Baudisch A, Haßfeld S et al (2018) Right ventricular function and mechanics in chemotherapy- and radiotherapy-naïve cancer patients. Int J Cardiovasc Imaging 34:1581

42. Aggeli C, Verveniotis A, Andrikopoulou E et al (2018) Echocardiographic features of PFOs and paradoxical embolism: a complicated puzzle. Int J Cardiovasc Imaging 34:1849

43. Piechnik SK, Jerosch-Herold M (2018) Myocardial T1 mapping and extracellular volume quantification: an overview of technical and biological confounders. Int J Cardiovasc Imaging 34:3-14. https://doi.org/10.1007/s10554-017-1235-7

44. Lin K, Suwa K, Ma H et al (2018) Variability of native T1 values: implication for defining regional myocardial changes using MRI. Int J Cardiovasc Imaging 34:1637-1645. https://doi.org/10.1007/ s10554-018-1371-8

45. Huang H-H, Huang C-Y, Chen C-N et al (2018) Automatic regional analysis of myocardial native $\mathrm{T} 1$ values: left ventricle segmentation and AHA parcellations. Int J Cardiovasc Imaging 34:131-140. https://doi.org/10.1007/s10554-017-1216-x

46. Lagan J, Schmitt M, Miller CA (2018) Clinical applications of multi-parametric CMR in myocarditis and systemic inflammatory diseases. Int J Cardiovasc Imaging 34:35-54. https://doi. org/10.1007/s10554-017-1063-9

47. Coelho-Filho OR, Shah R, Lavagnoli CFR et al (2018) Myocardial tissue remodeling after orthotopic heart transplantation: a pilot cardiac magnetic resonance study. Int J Cardiovasc Imaging 34:15-24. https://doi.org/10.1007/s10554-016-0937-6

48. Yuan Y, Cai J, Cui Y et al (2018) CMR-derived extracellular volume fraction $(\mathrm{ECV})$ in asymptomatic heart transplant recipients: correlations with clinical features and myocardial edema. Int $\mathbf{J}$ Cardiovasc Imaging 34:1959-1967. https://doi.org/10.1007/s1055 4-018-1421-2 
49. Nam BD, Kim SM, Jung HN et al (2018) Comparison of quantitative imaging parameters using cardiovascular magnetic resonance between cardiac amyloidosis and hypertrophic cardiomyopathy: inversion time scout versus T1 mapping. Int J Cardiovasc Imaging 34:1769-1777. https://doi.org/10.1007/s10554-018-1385-2

50. Kuijpers D, van Dijk R, van Assen M et al (2018) Disagreement between splenic switch-off and myocardial T1-mapping after caffeine intake. Int J Cardiovasc Imaging 34:625-632. https://doi. org/10.1007/s10554-017-1274-0

51. Gassenmaier S, van der Geest RJ, Schoepf UJ et al (2018) Quantitative inversion time prescription for myocardial late gadolinium enhancement using T1-mapping-based synthetic inversion recovery imaging: reducing subjectivity in the estimation of inversion time. Int J Cardiovasc Imaging 34:921-929. https:// doi.org/10.1007/s10554-017-1294-9

52. Jellis CL, Yingchoncharoen T, Gai N et al (2018) Correlation between right ventricular T1 mapping and right ventricular dysfunction in non-ischemic cardiomyopathy. Int J Cardiovasc Imaging 34:55-65. https://doi.org/10.1007/s10554-017-1113-3

53. Podlesnikar T, Delgado V, Bax JJ (2018) Cardiovascular magnetic resonance imaging to assess myocardial fibrosis in valvular heart disease. Int J Cardiovasc Imaging 34:97-112. https://doi. org/10.1007/s10554-017-1195-y

54. Avitzur N, Satriano A, Afzal M et al (2018) 3D myocardial deformation analysis from cine MRI as a marker of amyloid protein burden in cardiac amyloidosis: validation versus T1 mapping. Int J Cardiovasc Imaging 34:1937-1946. https://doi.org/10.1007/s1055 4-018-1410-5

55. Bhatti S, Vallurupalli S, Ambach S et al (2018) Myocardial strain pattern in patients with cardiac amyloidosis secondary to multiple myeloma: a cardiac MRI feature tracking study. Int J Cardiovasc Imaging 34:27-33. https://doi.org/10.1007/s10554-016-0998-6

56. van Everdingen WM, Zweerink A, Nijveldt R et al (2018) Comparison of strain imaging techniques in CRT candidates: CMR tagging, CMR feature tracking and speckle tracking echocardiography. Int J Cardiovasc Imaging 34:443-456. https://doi. org/10.1007/s10554-017-1253-5

57. Narang A, Blair JE, Patel MB et al (2018) Myocardial perfusion reserve and global longitudinal strain as potential markers of coronary allograft vasculopathy in late-stage orthotopic heart transplantation. Int J Cardiovasc Imaging 34:1607-1617. https:// doi.org/10.1007/s10554-018-1364-7

58. Liu B, Dardeer AM, Moody WE et al (2018) Reference ranges for three-dimensional feature tracking cardiac magnetic resonance: comparison with two-dimensional methodology and relevance of age and gender. Int J Cardiovasc Imaging 34:761-775. https://doi. org/10.1007/s10554-017-1277-x

59. Hwang J-W, Cha MJ, Kim SM et al (2018) Relationship between cardiovascular risk factors and myocardial strain values of both ventricles in asymptomatic Asian subjects: measurement using cardiovascular magnetic resonance tissue tracking. Int J Cardiovasc Imaging 34:1949-1957. https://doi.org/10.1007/s1055 4-018-1414-1

60. Suinesiaputra A, Sanghvi MM, Aung N et al (2018) Fully-automated left ventricular mass and volume MRI analysis in the UK Biobank population cohort: evaluation of initial results. Int $\mathbf{J}$ Cardiovasc Imaging 34:281-291. https://doi.org/10.1007/s1055 4-017-1225-9

61. Göransson C, Vejlstrup N, Carlsen J (2018) Reproducibility of peak filling and peak emptying rate determined by cardiovascular magnetic resonance imaging for assessment of biventricular systolic and diastolic dysfunction in patients with pulmonary arterial hypertension. Int J Cardiovasc Imaging 34:777-786. https://doi. org/10.1007/s10554-017-1281-1

62. Roberts PA, Lin ACW, Cowan BR et al (2018) Comparison of effects of losartan and metoprolol on left ventricular and aortic function at rest and during exercise in chronic aortic regurgitation. Int J Cardiovasc Imaging 34:615-624. https://doi.org/10.1007/ s10554-017-1268-y

63. Shang Y, Zhang X, Leng W et al (2018) Left atrium passive ejection fraction is the most sensitive index of type 2 diabetes mellitus-related cardiac changes. Int J Cardiovasc Imaging 34:141-151. https://doi.org/10.1007/s10554-017-1213-0

64. Stillman AE, Oudkerk M, Bluemke DA et al (2018) Imaging the myocardial ischemic cascade. Int J Cardiovasc Imaging 34:12491263. https://doi.org/10.1007/s10554-018-1330-4

65. Baritussio A, Scatteia A, Bucciarelli-Ducci C (2018) Role of cardiovascular magnetic resonance in acute and chronic ischemic heart disease. Int J Cardiovasc Imaging 34:67-80. https://doi. org/10.1007/s10554-017-1116-0

66. Sharif B, Motwani M, Arsanjani R et al (2018) Impact of incomplete ventricular coverage on diagnostic performance of myocardial perfusion imaging. Int J Cardiovasc Imaging 34:661-669. https://doi.org/10.1007/s10554-017-1265-1

67. Spiczak von J, Manka R, Gotschy A et al (2018) Fusion of CT coronary angiography and whole-heart dynamic 3D cardiac MR perfusion: building a framework for comprehensive cardiac imaging. Int J Cardiovasc Imaging 34:649-660. https://doi.org/10.1007/ s10554-017-1260-6

68. Nordin S, Dancy L, Moon JC, Sado DM (2018) Clinical applications of multiparametric CMR in left ventricular hypertrophy. Int J Cardiovasc Imaging 34:577-585. https://doi.org/10.1007/s1055 4-018-1320-6

69. Maragiannis D, Alvarez PA, Ghosn MG et al (2018) Left ventricular function in patients with hypertrophic cardiomyopathy and its relation to myocardial fibrosis and exercise tolerance. Int J Cardiovasc Imaging 34:121-129. https://doi.org/10.1007/s1055 4-017-1214-Z

70. Gommans DHF, Cramer GE, Bakker J et al (2018) High $\mathrm{T} 2$-weighted signal intensity for risk prediction of sudden cardiac death in hypertrophic cardiomyopathy. Int J Cardiovasc Imaging 34:113-120. https://doi.org/10.1007/s10554-017-1252-6

71. Cheng S, Choe YH, Ota $\mathrm{H}$ et al (2018) CMR assessment and clinical outcomes of hypertrophic cardiomyopathy with or without ventricular remodeling in the end-stage phase. Int J Cardiovasc Imaging 34:597-605. https://doi.org/10.1007/s10554-017-1263-3

72. Kamper L, Dreger NM, Brandt AS et al (2018) Diffusion-weighted MRI and PET-CT in the follow up of chronic periaortitis. Int J Cardiovasc Imaging 34:1779-1785. https://doi.org/10.1007/s1055 4-018-1395-0

73. Zhu C, Haraldsson H, Kallianos K et al (2018) Gated thoracic magnetic resonance angiography at 3T: noncontrast versus blood pool contrast. Int J Cardiovasc Imaging 34:475-483. https://doi. org/10.1007/s10554-017-1242-8

74. Kamphuis VP, Westenberg JJM, van der Palen RLF et al (2018) Scan-rescan reproducibility of diastolic left ventricular kinetic energy, viscous energy loss and vorticity assessment using 4D flow MRI: analysis in healthy subjects. Int J Cardiovasc Imaging 34:905-920. https://doi.org/10.1007/s10554-017-1291-z

75. Mukai K, Burris NS, Mahadevan VS et al (2018) 4D flow image quality with blood pool contrast: a comparison of gadofosveset trisodium and ferumoxytol. Int J Cardiovasc Imaging 34:273-279. https://doi.org/10.1007/s10554-017-1224-x

76. Wehrum T, Günther F, Kams M et al (2018) Quantification of aortic stiffness in stroke patients using 4D flow MRI in comparison with transesophageal echocardiography. Int J Cardiovasc Imaging 34:1629-1636. https://doi.org/10.1007/s10554-018-1369-2

77. Bech-Hanssen O, Svensson F, Polte CL et al (2018) Characterization of complex flow patterns in the ascending aorta in patients with aortic regurgitation using conventional phase-contrast velocity MRI. Int J Cardiovasc Imaging 34:419-429. https://doi. org/10.1007/s10554-017-1239-3 
78. Schäfer M, Morgan GJ, Mitchell MB et al (2018) Impact of different coarctation therapies on aortic stiffness: phase-contrast MRI study. Int J Cardiovasc Imaging 34:1459-1469. https://doi. org/10.1007/s10554-018-1357-6

79. Yamasaki Y, Kawanami S, Kamitani T et al (2018) Noninvasive quantification of left-to-right shunt by phase contrast magnetic resonance imaging in secundum atrial septal defect: the effects of breath holding and comparison with invasive oximetry. Int $\mathrm{J}$ Cardiovasc Imaging 34:931-937. https://doi.org/10.1007/s1055 4-018-1297-1

80. Shehu N, Meierhofer C, Messroghli D et al (2018) Diffuse fibrosis is common in the left, but not in the right ventricle in patients with transposition of the great arteries late after atrial switch operation. Int J Cardiovasc Imaging 34:1241-1248. https://doi.org/10.1007/ s10554-018-1338-9

81. Positano V, Meloni A, Macaione F et al (2018) Non-compact myocardium assessment by cardiac magnetic resonance: dependence on image analysis method. Int J Cardiovasc Imaging 34:12271238. https://doi.org/10.1007/s10554-018-1331-3

82. Schmidt EJ, Halperin HR (2018) MRI use for atrial tissue characterization in arrhythmias and for EP procedure guidance. Int J Cardiovasc Imaging 34:81-95. https://doi.org/10.1007/s 1055 4-017-1179-y

83. Zghaib T, Shahid A, Pozzessere C et al (2018) Validation of contrast-enhanced time-resolved magnetic resonance angiography in pre-ablation planning in patients with atrial fibrillation: comparison with traditional technique. Int J Cardiovasc Imaging 34:1451-1458. https://doi.org/10.1007/s10554-018-1355-8

84. Behm P, Gastl M, Jahn A et al (2018) CMR-guidance of passively tracked endomyocardial biopsy in an in vivo porcine model. Int $\mathrm{J}$ Cardiovasc Imaging 34:1917-1926. https://doi.org/10.1007/s1055 4-018-1402-5

85. Ghekiere O, Bielen J, Leipsic J, Dewilde W, Mancini I, Hansen D, Dendale P, Nchimi A (2018) Correlation of FFR-derived from CT and stress perfusion CMR with invasive FFR in intermediategrade coronary artery stenosis. Int J Cardiovasc Imaging. https:// doi.org/10.1007/s10554-018-1464-4

86. van Assen M, Pelgrim GJ, Slager E, van Tuijl S, Schoepf UJ, Vliegenthart R, Oudkerk M (2018) Low CT temporal sampling rates result in a substantial underestimation of myocardial blood flow measurements. Int J Cardiovasc Imaging. https://doi. org/10.1007/s10554-018-1451-9

87. Nozue T, Takamura T, Fukui K, Hibi K, Kishi S, Michishita I (2018) Assessment of factors associated with measurability of fractional flow reserve derived from coronary computed tomography angiography in type 2 diabetic patients with intermediate coronary artery stenosis. Int J Cardiovasc Imaging. https://doi. org/10.1007/s10554-018-1476-0

88. van der Werf NR, Willemink MJ, Willems TP, Vliegenthart R, Greuter MJW, Leiner T (2018) Influence of heart rate on coronary calcium scores: a multi-manufacturer phantom study. Int $\mathbf{J}$ Cardiovasc Imaging 34(6):959-966. https://doi.org/10.1007/s1055 4-017-1293-x
89. van der Werf NR, Willemink MJ, Willems TP, Greuter MJW, Leiner T (2018) Influence of iterative reconstruction on coronary calcium scores at multiple heart rates: a multivendor phantom study on state-of-the-art CT systems. Int J Cardiovasc Imaging 34(6):947-957. https://doi.org/10.1007/s10554-017-1292-y

90. Lindner S, Behnes M, Wenke A, Sartorius B, Ansari U, Akin M, Mashayekhi K, Vogler N, Haubenreisser H, Schoenberg SO, Borggrefe M, Akin I (2018) Assessment of peri-device leaks after interventional left atrial appendage closure using standardized imaging by cardiac computed tomography angiography. Int $\mathrm{J}$ Cardiovasc Imaging. https://doi.org/10.1007/s10554-018-1493-z

91. Obasare E, Mainigi SK, Morris DL, Slipczuk L, Goykhman I, Friend E, Ziccardi MR, Pressman GS (2018) CT based 3D printing is superior to transesophageal echocardiography for preprocedure planning in left atrial appendage device closure. Int J Cardiovasc Imaging 34(5):821-831. https://doi.org/10.1007/s1055 4-017-1289-6

92. Sims JR, Anavekar NS, Chandrasekaran K, Steckelberg JM, Wilson WR, Gersh BJ, Baddour LM, DeSimone DC (2018) Utility of cardiac computed tomography scanning in the diagnosis and pre-operative evaluation of patients with infective endocarditis. Int J Cardiovasc Imaging 34(7):1155-1163. https://doi.org/10.1007/ s10554-018-1318-0

93. Goo HW (2018) Coronary artery anomalies on preoperative cardiac CT in children with tetralogy of Fallot or Fallot type of double outlet right ventricle: comparison with surgical findings. Int J Cardiovasc Imaging 34(12):1997-2009. https://doi.org/10.1007/ s10554-018-1422-1

94. Xiang Y, Cheng G, Jin K, Zhang X, Yang Y (2018) Computed tomography findings and preoperative risk factors for mortality of total anomalous pulmonary venous connection. Int J Cardiovasc Imaging. https://doi.org/10.1007/s10554-018-1405-2

95. Gao X, Boccalini S, Kitslaar PH, Budde RPJ, Tu S, Lelieveldt BPF, Dijkstra J, Reiber JHC (2018) A novel software tool for semi-automatic quantification of thoracic aorta dilatation on baseline and follow-up computed tomography angiography. Int J Cardiovasc Imaging. https://doi.org/10.1007/s10554-018-1488-9

96. Pourmorteza A, Keller N, Chen R, Lardo A, Halperin H, Chen MY, McVeigh E (2018) Precision of regional wall motion estimates from ultra-low-dose cardiac CT using SQUEEZ. Int J Cardiovasc Imaging 34(8):1277-1286. https://doi.org/10.1007/s1055 4-018-1332-2

97. Abd Alamir M, Noack P, Jang KH, Moore JA, Goldberg R, Poon M (2018) Computer-aided analysis of 64- and 320-slice coronary computed tomography angiography: a comparison with expert human interpretation. Int J Cardiovasc Imaging 34(9):1473-1483. https://doi.org/10.1007/s 10554-018-1361-X

Publisher's Note Springer Nature remains neutral with regard to jurisdictional claims in published maps and institutional affiliations. 